\title{
Evaluating Interfaces to Publicly Available Environmental Information
}

\author{
Peter Mooney and Adam C. Winstanley \\ National Center for Geocomptation, \\ National University of Ireland Maynooth, \\ Co. Kildare, Ireland \\ \{peter.mooney, adam.winstanley\}@nuim.ie
}

\begin{abstract}
In this paper we detail a number of recommendations regarding how to evaluate interfaces to systems providing public access to environmental data and information. The primary focus is based upon web and Internet-based interfaces. These recommendations will assist data providers and information managers to evaluate the effectiveness of their current data access interfaces. The paper also outlines some guidelines on how to ensure these software interfaces are accessible to all citizens.
\end{abstract}

Keywords: Environmental Information, Web-Based Interfaces, Accessibility, OGC Specifications.

\section{Introduction}

Given the high profile of environmental issues today it is not surprising that many citizens search for environmental information and data using the Internet [1]. The purpose of such searches are varied. Examples include scientists carrying out research, policy makers searching for documents on environmental legislation, or members of the public searching for information on the local environment in which they live. These different purposes and user categories mean that data providers must adopt a layered approach to environmental data distribution and information provision. Each category of user has different data requirements. These requirements are broadly classified as follows:

- Environmental scientists will require access to the highest resolution raw data. They will require that the data is distributed in the most suitable format for the thematic area. For example, large meteorological datasets should be distributed in the World Meteorological Organization (WMO) GRIB Format.

- Policy makers, strategic environmental assessment (SEA) reporters, and other researchers may not require the highest resolution data. However they still require aggregated data accompanied by graphical representations. They will require the data be distributed in accessible software formats that can be imported into popular statistical and data analysis software tools . 
- Public users (those not fitting into either of the previous categories) will require simple aggregated information. This information should possibly be accompanied by additional information on severe or unusual environmental events. For example, if Ozone air quality levels go above recommended public health protection thresholds. Simple statistical visualisations (trend charts, histograms, normal distributions) should be provided. If the underlying data has a distinct geospatial component visualisations through geographical maps should be considered.

This paper provides a brief overview discussion on the key components that we feel should be included when developing interfaces for access to publicly accessible environmental data. In this paper we define publicly accessible environmental data as data and information which can be accessed by any citizen without that citizen having to pay a data access fee. Our recommendations are based on our experience in management and distribution of environmental data arising from scientific research programmes in Ireland funded by the Irish Environmental Protection Agency.

\section{Building the Interface to the Environmental Data}

New user-interface metaphors to geospatial data, such as NASA Earthwind, Microsoft TerraEarth, and Google Earth, have increased expectation levels from the general public and scientific community toward availability of geospatial data and associated web-based 'geo-services'. Often mapping and Geographical Information Systems (GIS) services are often beyond the IT expenditures of most organisations or not viewed as fitting into the intended audience profile for the organisation [2]. Consequently environmental information is often distributed in difficult-to-use software formats or throught complicated user-interfaces requiring scientific expertise to navigate. This adversely affects the dissemination of environmental information. The general public will only support the sometimes inconvenient, expensive, and controversial actions that must be taken to protect the environment if they have access to information [3]. To understand why different user-interfaces must be considered it is necessary to summarise the different categories of environmental data.

\subsection{Categories of Environmental Data}

Environmental data and information has several interesting characteristics. These characteristics present challenges to user-interface designers when providing this environmental information to the public. These characteristics are summarised as follows:

- Cumulative environmental effects: Where environmental effects, such as climate change, must be viewed over very long timescales;

- Large Volumes of Data: Environmental data resulting from the monitoring of rapidly changing phenonemum usually contain large volumes of information 
over short time periods. For example monitoring air pollution parameters is often carried out over five minute intervals on a continuous daily basis.

- Environmental data is often monitored over large geographical areas.

- Large number of parameters: Some environmental datasets contain a large number of inter-related variables. This is particularily evident in metrological datasets.

- Measurements at very low concentrations: In some environmental monitoring, such as water quality, some parameters are measured over a very small value scale.

- Public health information: Organisations that carry out air quality monitoring are required to highlight when various public health protection thresholds are exceeded.

The next two sections will describe various approaches to dealing with these categories of environmental data.

\subsection{Distribution of Data Using Traditional Web Techniques}

Using traditional web techniques involves using HTML in combination with graphical visualisations of the data and information in addition to linkages to download services for access to the raw environmental data. We recommend that this approach is best handled using a dynamic web-page generation approach. Users are presented with a simple web interface from which they select the parameters, time ranges, locations, or events they require information on. Graphical visualisations of these choices are then displayed, summary data or information is displayed, and links to the raw data used for the graphical visualisations. An example implementing these techniques is available on our greenhouse gas emissions inventory website http://coe.epa.ie/ghg. Figure1 shows the number of unique public user requests to an information resource on our web-server. For this particular resource (greenhouse gas emissions inventory data) public users were must more interesed in the visualisations of the dataset using graphical means through histograms, distributions, and trend graphs. The document formats (PDF and MS Word) were reasonably popular. However it is difficult to assertain what these documents were used for after they were downloaded. The Comma Separated Values (CSV) files available for download with the raw data from the graphical visualisations provided to be the most popular raw-data format. This is probably due to the fact that the CSV format is readily imported into most popular statistical packages.

\subsection{Distribution of Data Using OGC Web Map Services}

Environmental data monitored over large environmental areas or data that contains distinctly local environmental interest benefit from be distributed in a mapbased interfaces. The Open Geospatial Consortium (http://www.opengis.org) defines a Web Map Services (WMS) specifications. When OGC WMS are compared with traditional mapping processes the distribution of online mapping 


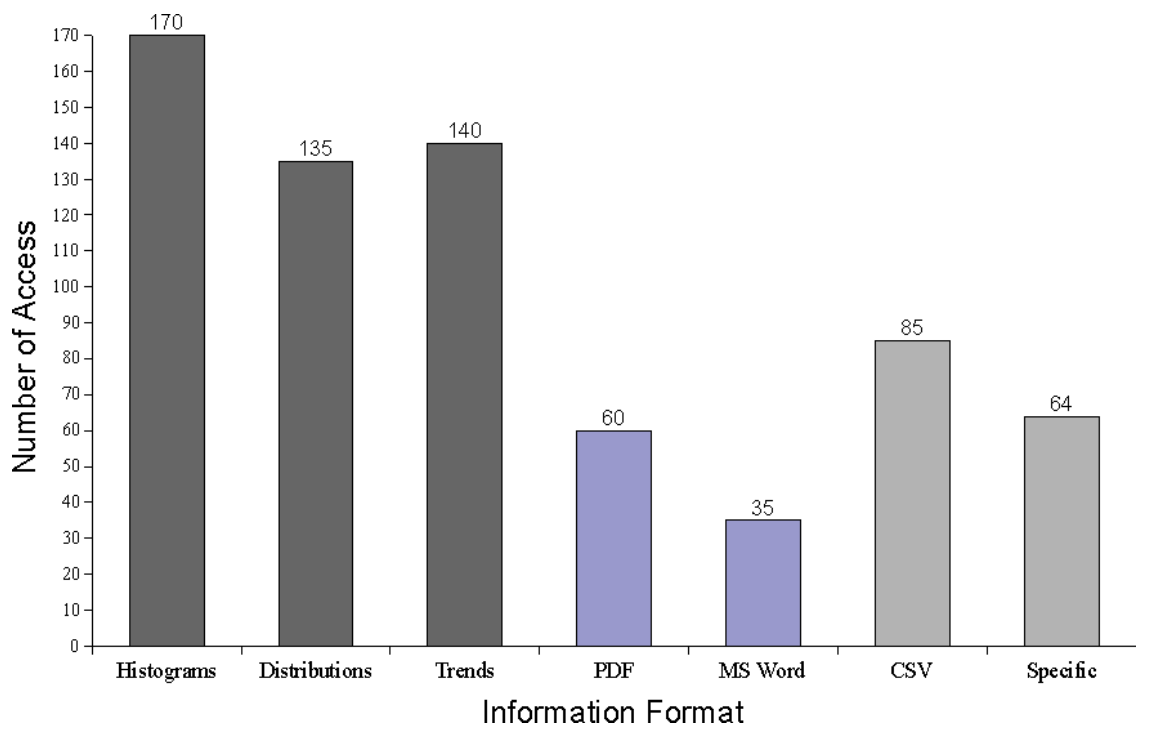

Fig. 1. Public Access to Various Information Formats

results are quicker, more easily customised, and more accessible to a greater number of people. No GIS or mapping skills are required on behalft of the end users. In line with the European INSPIRE Directive 4 the data management of the geospatial data is performed "as close to the source as possible". Providing OCG WMS access to the data allows all data and information be managed at one point. This reduces the possibility of duplication of data, and subsequent data quality issues as a result, while ensuring that the client user is always presented with the most current and available data. An example of ouput from an OGC WMS is displayed in Figure 2, This image was returned as the response to an WMS query. The user specified their options on a simple HTML web interface on a standard web-browser. The image depicts the Corine Land Cover Map for Europe 2000 (Wetlands Classification) layer with all towns in Ireland with population greater than 10,000 (square dots). This is a static PNG image returned to the user. A user may draw the conclusions that the majority of large towns in Ireland are situated away from areas of wetland environments. The two layers of geospatial data which are integrated together in this graphic were specified by the user using a combination of checkboxes and drop-down-lists to select which layers they wished to see combined.

A well-known example of OGC WMS being used for environmental information is at the website of the Environment Agency UK (EA-UK). The EA-UK have a "What's in my backyard?" [5] section where public users can search by postal code or town name for environmental information on that area. The example shows triangles beside rivers which have water quality standard targets set for them. 


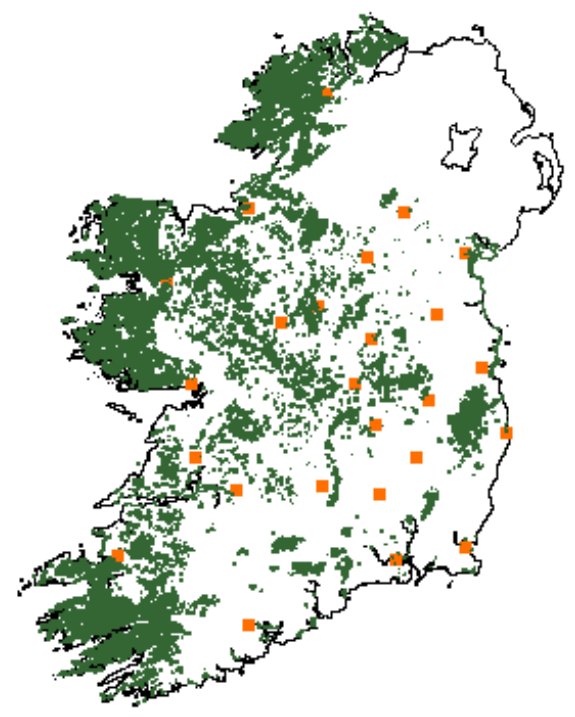

Fig. 2. An example of a simple static image returned from a OGC WMS query

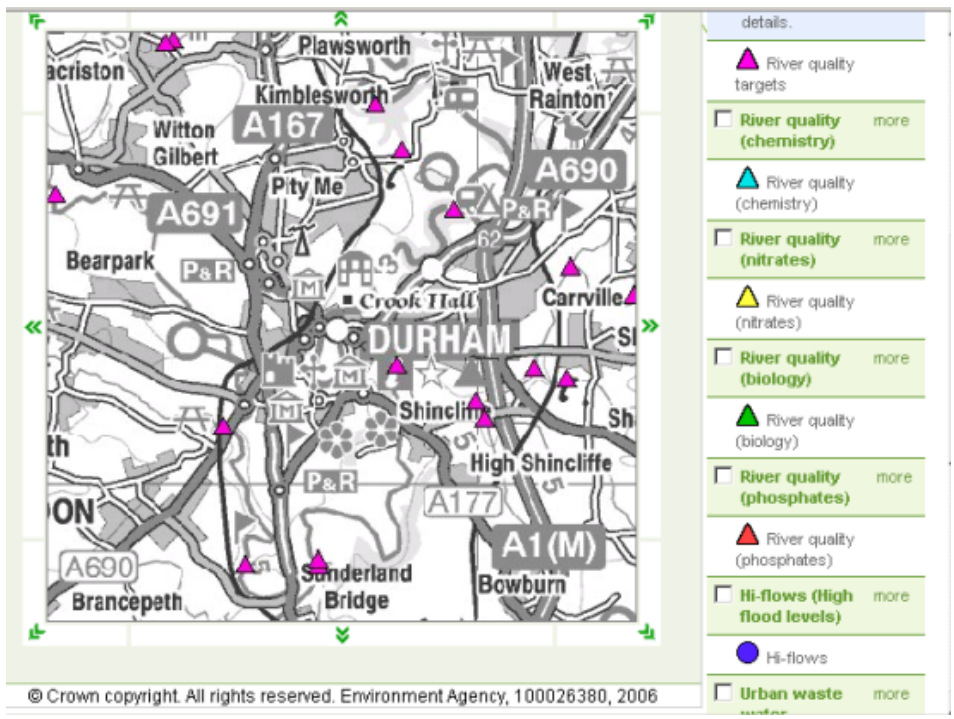

Fig. 3. An example map returned from a query for town of Durham on 5]

The list to the right-hand side of the map image allows users to enable the checkboxes of various other layers they wish to see on the map - for example river water quality in terms of biological status, or high flood level warnings. As 
in Figure 2 all of this functionality is available to the end user using at minimum a standard web-browser.

\section{Conclusions}

This paper has discussed in high level detail approaches to making environmental data and information accessible to the public. The key to successful distribution of these types of data is understanding the type of user audience. When the audience is spread across the wide spectrum of expertise in the general public the data access interfaces must cater equally well for all users. Discovery metadata about the data should be displayed in a simple format offering non-specialist users the opportunity to learn who produced the data, where it can be obtained, how it can be obtained and the costs associated with accessing the data (if there is a cost-recovery requirement involved). The ISO 19115 metadata standard provides for all of these discovery metadata items. The ISO 19115 metadata is stored in XML format and can be easily displayed in web interfaces and integrated into other data sources. There are a number of recommendations that we can make regarding web-based interfaces to publicly available environmental information:

1. Users should not be required to download any specialist software tools or packages to allow them to access the data or information. Current Internet browser software with basic configuration should be sufficient.

2. Web Accessibility Guidelines should be strictly adhered to[6]. In particular the following conditions should be available

- All web pages (dynamic and static) display graceful degradation as the viewing window is resized

- If the user does not have CSS support (or has it disabled) all pages providing publicly available content using CSS should be capable of being read sensibly in a linear fashion when CSS support is disabled.

- All hyperlinks should be properly titled using the title attribute in the HTML <a> anchor tag.

Interfaces to environmental data with a global dimension provide a very wide range of functionality for users. We have found that interfaces to environmental data on a more localised scale often have some serious problems associated with them. OGC WMS and other OGC specifications will allow smaller organisations to build better interfaces to their data inventories quickly at a low cost of investment.

We are also investigating the use of user-logging information to help guide our development of more useful web-interfaces to our environmental data. In the web-applications serving out our data we carefully log the actions taken by visitors to the web site. User actions that are logged include which pages they visit (in chronological order), which datasets they view and/or subsquently download, or which graphical representations they view most frequently. This 
information is stored directly in a relational database where an more indepth analysis of the behaviour of users visiting our web sites can be performed offline. In combination with web-server log files this is a valuable tool towards finding out what public visitors find useful, discovering which data services are most popular, and also highlighting any barriers inadvertently placed before users as they search for environmental data and information from our web services.

\subsection{Final Comments}

Public concern for environmental issues will continue to increase. This places even greater emphasis on data providers and information managers to make environmental information available in a timely fashion, without any technological barriers, and in the most suitable information representations. Computing power is still doubling every 18 months [7] and many public users now have own home computers more powerful than the servers of yesterday. Portable media device proliferation is difficult to avoid on a dialy basis. Today PDAs, Cell Phones, portable music players etc. are web and Internet enabled. Increasing numbers of citizens are using these devices to access information on the Internet. We feel that data and information managers must move from thinking in terms of static HTML web sites with collections of links to data file downloads to innovative means of data distribution. This data and information should be thought of in terms in "pieces of information" and "information packages" which can be integrated with other information. Devices other than PCs or workstations running Internet browser software can then pos-sibly consume these pieces of information. Environmental data and information useful to better public understanding of their environment should made be available from systems with open data access interfaces (ie those implementing the OGC Specifications). This should allow citizens to choose any client software which implements the OGC Specifications to access these data services. We believe that at minimum for environmental data with geospatial components the OGC WMS specifications should be implemented allowing users to access to simple map images [8] of the data. Public access to the data and information should not incur costs on the end-user. Some cost recovery mechanism may implemented for very rare datasets or datasets which are products or very expensive monitoring programmes. Younger Internet users of today have been brought up on a 'web-diet' rich in exciting user interface experiences (gmail, flickr, youTube, etc). When these users become adult users and consumers of environmental and geospatial information in the future they will expect these types of easy-to-use and access user interfaces as standard.

Acknowledgments. This work is being carried out in the Environmental Research Center (ERC) of the Irish Environmental Protection Agency (EPA). It is a postdoctoral research fellowship (2002-CC-FS) funded by ERTDI and the National Development Plan 


\section{References}

1. Schimak, G.: Environmental data management and monitoring system UWEDAT. Environmental Modelling And. Software 18(6), 573-580 (2003)

2. Maclachlan, J.C., Jerrett, M., Abernathy, T., Sears, M., Bunch, M.J.: Mapping health on the Internet: A new tool for environmental justice and public health research, Health And Place, Part Special Issue: Environmental Justice, Population Health, Critical Theory and GIS, March 2007, vol. 13(1), pp. 72-86 (2007)

3. Horinko, M.L.: Earth Observation Summit Conference, Keynote Address, Washington DC, USA (July 31, 2003) (Accessed February 07) http://yosemite. epa.gov/opa/admpress.nsf/

4. INSPIRE, Establishing An Infrastructure for Spatial Information in the European Community. PE-CONS 3685/2006, Brussels, Belguim (January 2007) (Accessed February 07) http://inspire.jrc.it

5. Environment Agency, What's in your backyard? The Environment Agency UK, (Accessed February 07) http://www.environment-ageny.gov.uk/maps/

6. Mooney, P., Winstanley, A.C.: Web Accessibility for Dynamically Generated Websites, IASTED Conference on Human Computer Interaction, Phoenix, Arizona, USA (November 2005)

7. Tuomi, I.: The Lives and Death of Moore's Law. First Monday, vol 7(11) (November 2002) (Accessed February 07) http://firstmonday.org/issues/ issue7_11/tuomi/index.html

8. Schutzberg, A. OGC Newsletter, January 2006. (Accessed February 07) http://www . opengeospatial.org/pressroom/newsletters/200601/ 